\title{
Lowering homocysteine levels may have no 'FAVORITable' effect on cardiovascular outcomes
}

Kidney transplant recipients often display an elevated plasma level of homocysteine, which has been shown to increase the risk of cardiovascular disease (CVD). Whether hyperhomocysteinemia is itself a cause of clinical events, or is instead a surrogate marker for CVD, is unknown. The FAVORIT trial assessed whether a reduction in total homocysteine levels using a vitamin B supplement containing folic acid would reduce the risk of CVD outcomes in kidney transplant recipients.

A total of 4,110 patients (who had stable kidney function and had undergone transplantation at least 6 months previously) were randomly assigned to receive a high dose of vitamin $\mathrm{B} 6(50 \mathrm{mg}$ ) and vitamin B12 (1 mg) with $5 \mathrm{mg}$ folic acid $(n=2,056)$, or to receive a low dose of vitamin B6 (1.4 mg) and vitamin B12 $(2 \mu \mathrm{g})$ without folic acid $(n=2,054)$. The primary outcome was a composite of incident or recurrent CVD events. Secondary outcomes included all-cause

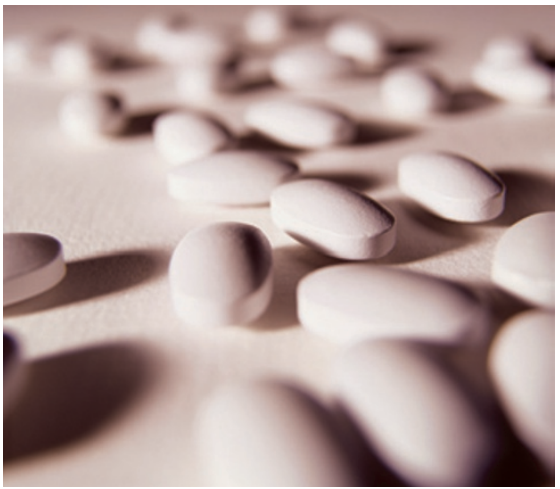

mortality and dialysis-dependent kidney failure. The study was stopped early after a mean of 4 years as the results had answered the original hypothesis.

The group receiving the high-dose supplement with folic acid showed a significantly greater reduction in homocysteine levels than did the low-dose group (mean 4-year change from baseline $-4.6 \mu \mathrm{mol} / \mathrm{l}$ versus $-0.2 \mu \mathrm{mol} / \mathrm{l}$ ). However, this change did not translate into improved outcomes as no differences were seen between groups in the occurrence of CVD outcomes, all-cause mortality, or dialysisdependent kidney failure. A similar number of patients in each group were hospitalized, and side effects did not differ between the groups. "Our findings confirm that there is no role for the screening and treatment of hyperhomocysteinemia in chronic kidney disease," says investigator Andrew Bostom. "Observational analyses of the FAVORIT trial database will be useful for generating new hypotheses regarding other potential risk factors for cardiorenal outcomes in transplant patients with chronic kidney disease."

Helene Myrvang

Original article Bostom, A. G. et al. Homocysteine-lowering and cardiovascular disease outcomes in kidney transplant recipients: primary results from the Folic Acid for Vascular Outcome Reduction in Transplantation trial. Circulation doi:10.1161/CIRCULATIONAHA.110.000588 